\title{
Stability theory and existence of solution to a multi-point boundary value problem of fractional differential equations
}

\author{
Muhammad Zamir ${ }^{1} \cdot$ Kamal Shah $^{2} \cdot$ Muhammad Ikhlaq Chohan $^{3}$
}

Received: 21 January 2019 / Accepted: 21 February 2019 / Published online: 2 March 2019

(c) The Author(s) 2019

\begin{abstract}
The aims and objectives of this manuscript are concerned with the investigation of some appropriate conditions to establish existence theory of solutions to a class of nonlinear four-point boundary value problem (BVP) corresponding to fractional order differential equations (FODEs) provided as

$\left\{\begin{array}{l}{ }^{c} \mathscr{D}^{\omega} y(t)=\mathcal{F}\left(t, y(t),{ }^{c} \mathscr{D}^{\omega-1} y(t)\right), 1<\omega \leq 2, t \in \mathbf{J}=[0,1], \\ y(0)=\zeta y(\alpha), y(1)=\xi y(\beta), \xi, \zeta, \alpha, \beta \in(0,1),\end{array}\right.$

where ${ }^{c} \mathscr{D}^{\omega}$ is Caputo's fractional derivative of order $q$ and $\mathcal{F} \in(\mathbf{J} \times \mathbf{R} \times \mathbf{R}, \mathbf{R})$ may be nonlinear. The required conditions are obtained by using classical results of functional analysis and fixed point theory. Further, we establish some adequate conditions for the Ulam-Hyers stability and generalized Ulam-Hyers stability for the solutions to the considered BVP of nonlinear FODEs. We include a proper problem to illustrate our established results.
\end{abstract}

Keywords FODEs $\cdot$ Four-point boundary conditions $\cdot$ Ulam-Hyers stability (UHS)

Mathematics Subject Classification $26 \mathrm{~A} 33 \cdot 34 \mathrm{~A} 08 \cdot 35 \mathrm{~B} 40$

\section{Introduction}

FODEs are used to model complex phenomena of physically significant problems arising from indifferent areas such as physics, engineering and other applied disciplines, and there is a broad set of applications. Due to the large numbers of applications of FODEs in sciences and engineering,

Kamal Shah

kamalshah408@gmail.com; kamal@uom.edu.pk

Muhammad Zamir

zamirburqi@yahoo.com

Muhammad Ikhlaq Chohan

chohan@buc.edu.om

1 Department of Mathematics, University of Science and Technology, Banuu, Khyber Pakhtunkhwa, Pakistan

2 Department of Mathematics, University of Malakand, Dir (L), Khyber Pakhtunkhwa, Pakistan

3 Department of Business Administration and Accounting, Al Buraimi University College, Muscat, Al-Buraimi, Sultanate of Oman plenty of research papers have been written in this area. As a result, the theory of FODEs has emerged as an important area of investigation in recent years [1,2]. Valuable contribution has been done dealing with the qualitative theory and numerical analysis of solutions to initial and boundary value problems (BVPs) of nonlinear FODEs. The researchers have given much attention on qualitative theory of solutions for mentioned FODEs [3-12], and references therein. Since BVPs arise in various disciplines of physics, engineering and in dynamics, etc., from applications point of view, here we refer some famous BVPs of differential equations which are the wave equation, like the computation of the normal modes, the Sturm-Liouville problems and Dirichlet problem, etc., see [13-17]. For usability purposes, a BVP should be well posed which implies that a unique solution exists corresponding to the input which depends continuously on the input . In thermal sciences BVPs have significant applications, for instance, to find the temperature at all points of an iron bar with one end kept at lowest energy level and the other end at the freezing point of water. Due to these importance applications, researchers studied BVPs 
of both classical and arbitrary order differential equations from different aspects. One of the important aspects which has been greatly developed and well explored by different researchers is known as existence theory. The respective aspects have been explored for BVPs of FODEs, see for some detail [18-22].

In the last few years, nonlinear BVPs of FODEs were investigated increasingly. For instance, in [22], Benchora and his co-authors investigated the following anti-periodic BVP given by

$\left\{\begin{array}{l}{ }^{c} \mathscr{D}^{\omega} y(t)=\Phi\left(t, y(t),{ }^{c} \mathscr{D}^{\omega-1} y(t)\right), \quad t \in[0, b], \\ y(0)=-y(b), y^{\prime}(0)=-y^{\prime}(b),\end{array}\right.$

where $1<\omega \leq 2, \Phi \in([0, b] \times \mathbf{R} \times \mathbf{R}, \mathbf{R})$.

In last few decades another important aspect which has greatly investigated for the solutions of differential, integral and functional equations is known as stability analysis. The mentioned aspects is very important from numerical and optimization point of view. This is due to the fact that most of the nonlinear problems of fractional calculus and applied analysis are quite difficult to solve for actual solution. In such a situation, one need approximate solutions which are near the actual solution of the corresponding problem. In the mentioned situation, stability of the solutions is necessary. Researchers investigated different kinds of stability to differential, integral and functional equations like exponential, Mittag-Leffler and Lyapunov stability, for detail see [23-25]. Recently, some authors explored the another form of stability known as Ulam-Hyers and generalized Ulam-Hyers stability for the solutions of FODEs, see [24, 26-29]. The aforesaid stability has been very well studied for initial value problems and simple two-point BVPs of linear and nonlinear FODEs, see [30-32]. The concerned stability is very rarely investigated for the three- or more point BVPs of FODEs. Here we remark that nonlocal BVPs of FODEs are of key importance for engineers, physics, etc. The stable solutions of the aforesaid problems help us in understanding the phenomenon which has the differential equations.

Therefore, inspired from the aforementioned work and importance, here we investigate the aforesaid analysis to a four-point BVP of nonlinear FODEs suggested as

$$
\left\{\begin{array}{l}
{ }^{c} \mathscr{D}^{\omega} y(t)=\mathcal{F}\left(t, y(t),{ }^{c} \mathscr{D}^{\omega-1} y(t)\right), \quad t \in \mathbf{J}, \\
y(0)=\zeta y(\alpha), y(1)=\xi y(\beta),
\end{array}\right.
$$

where ${ }^{c} \mathscr{D}^{\omega}$ is the Caputo derivative of order $\omega \in(1,2]$, $\mathcal{F} \in(\mathbf{J} \times \mathbf{R} \times \mathbf{R}, \mathbf{R})$ is continuous function and the parameters satisfy $0 \leq \zeta, \xi \leq 1 \alpha, \beta \in(0,1)$ such that $((1-\zeta)(1-\xi \beta)+\zeta \alpha(1-\xi)) \neq 0$. With the help of Banach and Schauder fixed point theorem we develop our required results for the existence and uniquness of solution to the considered problem. The stability results are useful consequences of the existence theory and can be obtained by applying classical functional analysis. We include an example to illustrate our results.

\section{Preliminaries}

Since the space $C(\mathbf{J}, \mathbf{R})$ is a Banach space endowed with a norm $\|y\|_{\infty}=\sup \{|y|: t \in \mathbf{J}\}, L^{1}(\mathbf{J}, \mathbf{R})$ for the space of Lebesgue integrable functions defined on $\mathbf{J}$ which is a Banach space corresponding to the norm $\|y\|_{L^{1}}=\int_{0}^{1}|y(t)| \mathrm{d} t$. The space defined as

$\widetilde{C}(\mathbf{J}, \mathbf{R})=\left\{y \in C(\mathbf{J}, \mathbf{R}):{ }^{c} \mathscr{D}^{\omega-1} y \in C(\mathbf{J}, \mathbf{R})\right\}$, endowed with a norm $\|y\|_{\tilde{C}}=\max \left\{\|y\|_{\infty},\left\|\mathscr{D}^{\omega-1} y\right\|_{\infty}\right\}$ is a Banach space [22]. We provide some basic results and definitions.

Definition 2.1 ([1]). The integral with fractional order $\omega>0$ for a function $g \in L^{1}(\mathbf{J}, \mathbf{R})$ is recalled by

$\mathscr{I}^{\omega} g(t)=\frac{1}{\Gamma(\omega)} \int_{0}^{t}(t-\theta)^{\omega-1} g(\theta) \mathrm{d} \theta$.

Definition 2.2 ([1]). The Caputo derivative of order $\omega>0$ of a function $g(t)$ on $(0, \infty)$ is defined by

$\left({ }^{c} \mathscr{D}^{\omega} g\right)(t)=\frac{1}{\Gamma(n-\omega)} \int_{0}^{t}(t-\theta)^{n-\omega-1} g^{(n)}(\theta) \mathrm{d} \theta$,

where $n=[\omega]+1$.

Lemma 2.3 ([2]). The FODE with order $\omega>0$ given by

${ }^{c} \mathscr{D}^{\omega} y(t)=0, n-1<\omega \leq n$,

has a unique solution provided as

$y(t)=b_{0}+b_{1} t+b_{2} t^{2}+\cdots+b_{n-1} t^{n-1}$, where $b_{i} \in \mathbf{R}$,

$i=0,1,2, \ldots, n-1, n=[\omega]+1$.

Lemma 2.4 ([2]) For $\omega>0$,

$\mathscr{I}^{\omega} \mathscr{D}^{\omega} g(t)=g(t)+b_{0}+b_{1} t+b_{2} t^{2}+\cdots+b_{n-1} t^{n-1}$,

where $b_{i} \in \mathbf{R}, i=0,1,2, \ldots, n-1, n=[\omega]+1$ holds.

The next lemma plays an important role for converting BVPs to integral equations.

Lemma 2.5 For $g \in C(\mathbf{J}, \mathbf{R})$, the linear fractional order $B V P$

${ }^{c} \mathscr{D}^{\omega} y(t)=g(t), \quad t \in \mathbf{J}, \quad 1<\omega \leq 2$,

$y(0)=\zeta y(\alpha), y(1)=\xi y(\beta)$, 
has unique solution $y(t)=\int_{0}^{1} \mathscr{G}(t, \theta) g(\theta) \mathrm{d} \theta, t \in \mathbf{J}$, where

$$
\mathscr{G}(t, \theta)=\frac{1}{\Gamma(\omega)}\left\{\begin{array}{l}
(t-\theta)^{\omega-1}+\zeta \Delta(1-\xi \beta-(1-\xi) t)(\alpha-\theta)^{\omega-1}+\Delta \xi(\zeta \alpha+(1-\zeta) t)(\beta-\theta)^{\omega-1} \\
\quad-\Delta(\zeta \alpha+(1-\zeta) t)(1-\theta)^{\omega-1}, \quad \text { if } 0 \leq \theta \leq \min \{t, \alpha, \beta\} \leq 1, \\
(t-\theta)^{\omega-1}+\Delta \xi(\zeta \alpha+(1-\zeta) t)(\beta-\theta)^{\omega-1}-\Delta(\zeta \alpha+(1-\zeta) t)(1-\theta)^{\omega-1}, \\
\quad \text { if } 0 \leq \alpha \leq \theta \leq \min \{t, \beta\} \leq 1, \\
(t-\theta)^{\omega-1}-\Delta(\zeta \alpha+(1-\zeta) t)(1-\theta)^{\omega-1}, \quad \text { if } 0 \leq \max \{\alpha, \beta\} \leq \theta \leq t \leq 1, \\
\Delta \zeta(1-\xi \beta-(1-\xi) t)(\alpha-\theta)^{\omega-1}+\Delta \xi(\zeta \alpha+(1-\zeta) t)(\beta-\theta)^{\omega-1} \\
\Delta(\zeta \alpha+(1-\zeta) t)(1-\theta)^{\omega-1}, \quad \text { if } 0 \leq t \leq \theta \leq \min \{\alpha, \beta\} \leq 1, \\
\Delta \xi(\zeta \alpha+(1-\zeta) t)(\beta-\theta)^{\omega-1}-\Delta(\zeta \alpha+(1-\zeta) t)(1-\theta)^{\omega-1}, \\
\quad \text { if } 0 \leq \max \{t, \alpha\} \leq \theta \leq \beta \leq 1, \\
\Delta \zeta(1-\xi \beta-(1-\xi) t)(\alpha-\theta)^{\omega-1}-\Delta(\zeta \alpha+(1-\zeta) t)(1-\theta)^{\omega-1}, \\
\quad \text { if } 0 \leq \max \{t, \alpha, \beta\} \leq \theta \leq 1,
\end{array}\right.
$$

is Green's function, where $\Delta=((1-\zeta)(1-\xi \beta)+$ $\zeta \alpha(1-\xi))^{-1}$.

Proof Applying $\mathscr{I}^{\omega}$ on (2) and thanking to Lemma 2.4, we obtain

$y(t)=\mathscr{I}^{q} g(t)+b_{0}+b_{1} t, \quad t \in \mathbf{J}$.

The boundary condition $y(0)=\zeta y(\alpha)$ implies

$$
\begin{aligned}
b_{0}= & \frac{\Delta \zeta(1-\xi \beta)}{\Gamma(\omega)} \int_{0}^{\alpha}(\alpha-\theta)^{\omega-1} g(\theta) \mathrm{d} \theta \\
& +\frac{\Delta \zeta \xi \alpha}{\Gamma(\omega)} \int_{0}^{\beta}(\beta-\theta)^{\omega-1} g(\theta) \mathrm{d} \theta \\
& -\frac{\Delta \zeta \alpha}{\Gamma(\omega)} \int_{0}^{1}(1-\theta)^{\omega-1} g(\theta) \mathrm{d} \theta
\end{aligned}
$$

and the boundary condition $y(1)=\xi y(\beta)$ yields

$$
\begin{aligned}
b_{1}= & \frac{\Delta \xi(1-\zeta)}{\Gamma(\omega)} \int_{0}^{\beta}(\beta-\theta)^{\omega-1} g(\theta) \mathrm{d} \theta \\
& -\frac{\Delta \zeta(1-\xi)}{\Gamma(\omega)} \int_{0}^{\alpha}(\alpha-\theta)^{q-1} g(\theta) \mathrm{d} \theta \\
& -\frac{\Delta(1-\zeta)}{\Gamma(\omega)} \int_{0}^{1}(1-\theta)^{\omega-1} g(\theta) \mathrm{d} \theta .
\end{aligned}
$$

It follows from (4) that solution of the BVP (2) is

$$
\begin{aligned}
y(t)= & \frac{1}{\Gamma(\omega)} \int_{0}^{t}(t-\theta)^{\omega-1} g(\theta) \mathrm{d} \theta \\
& +\frac{\Delta(1-\xi \beta) \zeta-\Delta(1-\xi) \zeta t}{\Gamma(\omega)} \int_{0}^{\alpha}(\alpha-\theta)^{\omega-1} g(\theta) \mathrm{d} \theta \\
& +\frac{\Delta \zeta \alpha \xi+\Delta(1-\zeta) \xi t}{\Gamma(\omega)} \int_{0}^{\beta}(\beta-\theta)^{\omega-1} g(\theta) \mathrm{d} \theta \\
& -\frac{\Delta \zeta \alpha+\Delta(1-\zeta) t}{\Gamma(\omega)} \int_{0}^{1}(1-\theta)^{\omega-1} g(\theta) \mathrm{d} \theta \\
= & \int_{0}^{1} \mathscr{G}(t, \theta) g(\theta) \mathrm{d} \theta .
\end{aligned}
$$

\section{Existence of at least one solution: main result}

This part of the manuscript is devoted to study existence and uniqueness of solutions for the considered problem (1). For the required results, we use Schauder's fixed point theorem [33] and Banach contraction principle. Thanks to Lemma 2.5, the proposed problem (1) is equivalent to the given integral equation

$y(t)=\int_{0}^{1} \mathscr{G}(t, \theta) \mathcal{F}\left(\theta, y(\theta),{ }^{c} \mathscr{D}^{\omega-1} y(t)\right) \mathrm{d} \theta, \quad t \in \mathbf{J}$.

Let $N: \widetilde{C}(\mathbf{J}, \mathbf{R}) \rightarrow \widetilde{C}(\mathbf{J}, \mathbf{R})$ be the operator defined as

$N y(t)=\int_{0}^{1} \mathscr{G}(t, \theta) \mathcal{F}\left(\theta, y(\theta),{ }^{c} \mathscr{D}^{\omega-1} y(\theta)\right) \mathrm{d} \theta$, 
then by solutions of the BVP (1) we mean fixed points of $N$. Further, we need the following result for onward analysis.

$$
\begin{aligned}
& { }^{c} \mathscr{D}^{\omega-1} N y(t)=\int_{0}^{t} \mathcal{F}\left(\theta, y(\theta),{ }^{c} \mathscr{D}^{\omega-1} y(\theta)\right) \mathrm{d} \theta-\frac{\Delta t^{2-\omega}}{\Gamma(\omega) \Gamma(3-\omega)} \\
& {\left[\zeta(1-\xi) \int_{0}^{\alpha}(\alpha-\theta)^{\omega-1} \mathcal{F}\left(\theta, y(\theta),{ }^{c} \mathscr{D}^{\omega-1} y(\theta)\right) \mathrm{d} \theta\right.} \\
& +\xi(1-\zeta) \int_{0}^{\beta}(\beta-\theta)^{\omega-1} \mathcal{F}\left(\theta, y(\theta),{ }^{c} \mathscr{D}^{\omega-1} y(\theta)\right) \mathrm{d} \theta-(1-\zeta) \\
& \left.\int_{0}^{1}(1-\theta)^{\omega-1} \mathcal{F}\left(\theta, y(\theta),{ }^{c} \mathscr{D}^{\omega-1} y(\theta)\right) \mathrm{d} \theta\right] .
\end{aligned}
$$

Theorem 3.1 Assume that $\mathcal{F}: \mathbf{J} \times \mathbf{R} \times \mathbf{R} \rightarrow \mathbf{R}$ is continuous.

(i) There exist $p: \mathbf{J} \rightarrow \mathbf{R}^{+}$and $\psi:[0, \infty) \rightarrow(0, \infty)$ continuous and nondecreasing with

$|\mathcal{F}(t, y, z)| \leq p(t) \psi(|z|)$, for $t \in \mathbf{J}$ and each $y, z \in \mathbf{R}$.

(ii) There exists a constant $r>0$ satisfying

$r \geq \max \left\{\psi(r) p^{*} \mathscr{G}^{*}, \frac{p^{*} \psi(r)\left(\Gamma(\omega+1) \Gamma(3-\omega)+\Delta \zeta(1-\zeta) \alpha^{\omega}+\Delta \xi(1-\zeta) \beta^{\omega} \Delta(1-\zeta)\right)}{\Gamma(\omega+1) \Gamma(3-\omega)}\right\}$,

where $\quad p^{*}=\sup \{p(t), t \in J\}, \mathscr{G}^{*}=\sup _{t \in \mathbf{J}}$ $\int_{0}^{1}|\mathscr{G}(t, \theta)| \mathrm{d} \theta$.

Then, the suggested FODE (1) has at least one solution with $|y(t)| \leq r$ on $\mathbf{J}$.

Proof To prove the continuity of the operator $N$ defined in (6), we consider a sequence $\left\{y_{n}\right\}$ such that $y_{n} \rightarrow y$ in $\widetilde{C}(\mathbf{J}, \mathbf{R})$. Then, there exists $r>0$ such that for $\left\|y_{n}\right\|_{\tilde{C}} \leq r,\|y\|_{\tilde{C}} \leq r$, we get

$$
\begin{gathered}
\left|\left(N y_{n}\right)(t)-(N y)(t)\right| \leq \int_{0}^{1}|G(t, \theta)| \mid \mathcal{F}\left(\theta, y_{n}(\theta),\right. \\
\left.{ }^{c} \mathscr{D}^{\omega-1} y_{n}(\theta)\right)-f\left(\theta, y(\theta),{ }^{c} \mathscr{D}^{\omega-1} y(\theta)\right) \mid \mathrm{d} \theta,
\end{gathered}
$$

due to the continuity of $\mathcal{F}$ and Lebesgue dominated convergence theorem implies that

$\left\|N y_{n}-N y\right\|_{\infty} \rightarrow 0, n \rightarrow \infty$

Moreover

$$
\begin{aligned}
& \left|\left({ }^{c} \mathscr{D}^{\omega-1} N y_{n}\right)(t)-\left({ }^{c} \mathscr{D}^{\omega-1} N y\right)(t)\right| \leq \int_{0}^{t} \mid \mathcal{F}\left(\theta, y_{n}(\theta),\right. \\
& \left.\quad{ }^{c} \mathscr{D}^{\omega-1} y_{n}(\theta)\right)-\mathcal{F}\left(\theta, y(\theta),{ }^{c} \mathscr{D}^{\omega-1} y(\theta)\right) \mid \mathrm{d} \theta \\
& \quad+\frac{\Delta t^{2-\omega}}{\Gamma(\omega) \Gamma(3-\omega)}\left(\zeta(1-\xi) \int_{0}^{\alpha}(\alpha-\theta)^{\omega-1} \mid \mathcal{F}\left(\theta, y_{n}(\theta),\right.\right. \\
& \left.\quad{ }^{c} \mathscr{D}^{\omega-1} y_{n}(\theta)\right)-\mathcal{F}\left(\theta, y(\theta),{ }^{c} \mathscr{D}^{\omega-1} y(\theta)\right) \mid \mathrm{d} \theta \\
& \quad+\xi(1-\zeta) \int_{0}^{\beta}(\beta-\theta)^{\omega-1} \mid \mathcal{F}\left(\theta, y_{n}(\theta),{ }^{c} \mathscr{D}^{\omega-1} y_{n}(\theta)\right) \\
& \quad-\mathcal{F}\left(\theta, y(\theta),{ }^{c} \mathscr{D}^{\omega-1} y(\theta)\right) \mid \mathrm{d} \theta \\
& \quad+(1-\zeta) \int_{0}^{1}(1-\theta)^{\omega-1} \mid \mathcal{F}\left(\theta, y_{n}(\theta),{ }^{c} \mathscr{D}^{\omega-1} y_{n}(\theta)\right) \\
& \left.\quad-\mathcal{F}\left(\theta, y(\theta),{ }^{c} \mathscr{D}^{\omega-1} y(\theta)\right) \mid \mathrm{d} \theta\right),
\end{aligned}
$$

and again from the continuity of $\mathcal{F}$ and Lebesgue dominated convergence theorem, we get

$\left\|{ }^{c} \mathscr{D}^{\omega-1} N y^{n}-{ }^{c} \mathscr{D}^{\omega-1} N y\right\|_{\infty} \rightarrow 0$, as $n \rightarrow \infty$.
Now, we show that $N(D) \subseteq D$, where $D=\left\{y \in \widetilde{C}(\mathbf{J}, \mathbf{R}):\|y\|_{\widetilde{C}} \leq r\right\}$ is a closed and convex subset of $\widetilde{C}(\mathbf{J}, \mathbf{R})$. Take $y \in D$ and using the two conditions of Theorem 3.1, we obtain

$$
\begin{aligned}
|(N y)(t)| \leq & \int_{0}^{1}|\mathscr{G}(t, \theta)|\left|\mathcal{F}\left(\theta, y(\theta),{ }^{c} \mathscr{D}^{\omega-1} y(\theta)\right)\right| \mathrm{d} \theta \\
\leq & \psi\left(\|y\|_{\widetilde{C}}\right) p^{*} \mathscr{G}^{*}, \\
\left.\mid{ }^{c} \mathscr{D}^{\omega-1} N y\right)(t) \mid \leq & \frac{p^{*} \psi\left(\|y\|_{\widetilde{C}}\right)}{\Gamma(\omega+1) \Gamma(3-\omega)}(\Gamma(\omega+1) \Gamma(3-\omega) \\
& \left.+\mathrm{d} \zeta(1-\xi) \alpha^{\omega}+\Delta \xi(1-\zeta) \beta^{\omega}+\Delta(1-\zeta)\right) .
\end{aligned}
$$

Hence, it follows that

$\|N y\|_{\widetilde{C}} \leq r$ implies $N(D) \subseteq D$.

Finally, we prove that $N$ maps $D$ into an equi-continuous set of $\widetilde{C}(J, \mathbf{R})$. Consider $\tau_{1}<\tau_{2} \in \mathbf{J}$ and $y \in D$, then 


$$
\begin{aligned}
\left|(N y)\left(\tau_{2}\right)-(N y)\left(\tau_{1}\right)\right| \leq & \int_{0}^{1} \mid \mathscr{G}\left(\tau_{2}, \theta\right)-\mathscr{G}\left(\tau_{1}, \theta\right) \\
& \| \mathcal{F}\left(\theta, y(\theta),{ }^{c} \mathscr{D}^{\omega-1} y(\theta)\right) \mid \mathrm{d} \theta \\
& \leq p^{*} \psi(r) \int_{0}^{1}\left|\mathscr{G}\left(\tau_{2}, \theta\right)-\mathscr{G}\left(\tau_{1}, \theta\right)\right| \mathrm{d} \theta
\end{aligned}
$$

and

$$
\begin{aligned}
& \left|\left({ }^{c} \mathscr{D}^{\omega-1} N y\right)\left(\tau_{2}\right)-\left({ }^{c} \mathscr{D}^{\omega-1} N y\right)\left(\tau_{1}\right)\right| \leq p^{*} \psi(r) \\
& \quad\left[\tau_{2}-\tau_{1}+\frac{\Delta}{\Gamma(\omega+1) \Gamma(3-\omega)}\left(\zeta(1-\xi)\left(\tau_{2}^{2-\omega}-\tau_{1}^{2-\omega}\right) \alpha^{\omega}\right.\right. \\
& \left.\left.\quad+\xi(1-\zeta)\left(\tau_{2}^{2-\omega}-\tau_{1}^{2-\omega}\right) \beta^{\omega}+(1-\zeta)\left(\tau_{2}^{2-\omega}-\tau_{1}^{2-\omega}\right)\right)\right] .
\end{aligned}
$$

From the continuity of $\mathscr{G}$, it follows that $\mid(N y)\left(\tau_{2}\right)-$ $(N y)\left(\tau_{1}\right)|\rightarrow 0|,\left({ }^{c} \mathscr{D}^{\omega-1} N y\right)\left(\tau_{2}\right)-\left({ }^{c} \mathscr{D}^{\omega-1} N y\right)\left(\tau_{1}\right) \mid \rightarrow 0$ as $\tau_{1} \rightarrow \tau_{2}$. By Arzelà-Ascoli Theorem, $N$ is completely

$$
\begin{aligned}
&\left|{ }^{c} \mathscr{D}^{\omega-1}(N y)(t)-{ }^{c} \mathscr{D}^{\omega-1}(N \bar{y})(t)\right| \\
& \leq \frac{k}{\Gamma(\omega+1) \Gamma(3-\omega)}(\Gamma(\omega+1) \Gamma(3-\omega) \\
&\left.+\Delta \xi(1-\zeta) \beta^{\omega}+\Delta(1-\zeta)+\Delta \zeta(1-\xi) \alpha^{\omega}\right) \\
& \times\left(\|y-\bar{y}\|_{\infty}+\left\|^{c} \mathscr{D}^{\omega-1} y-{ }^{c} \mathscr{D}^{\omega-1} \bar{y}\right\|_{\infty}\right),
\end{aligned}
$$

which implies that

$$
\begin{aligned}
\|{ }^{c} & \mathscr{D}^{\omega-1} N y-{ }^{c} \mathscr{D}{ }^{\omega-1} N \bar{y} \|_{\infty} \\
& \leq \frac{2 k}{\Gamma(\omega+1) \Gamma(3-\omega)}(\Gamma(\omega+1) \Gamma(3-\omega) \\
& \left.+\Delta \xi(1-\zeta) \beta^{\omega}+\Delta(1-\zeta)+\Delta \zeta(1-\xi) \alpha^{\omega}\right) \times\|y-\bar{y}\|_{\widetilde{C}}
\end{aligned}
$$

From the relations (11) and (12), it follows that

$\|N y-N \bar{y}\|_{\widetilde{C}} \leq \max \left\{2 \mathscr{G}^{*} k, \frac{2 k\left(\Gamma(\omega+1) \Gamma(3-\omega)+\Delta \xi(1-\zeta) \beta^{\omega}+\Delta(1-\zeta)+\Delta \zeta(1-\xi) \alpha^{\omega}\right)}{\Gamma(\omega+1) \Gamma(3-\omega)}\right\}\|y-\widetilde{y}\|_{\tilde{C}}$,

continuous, and hence, by Schauder's fixed point theorem $N$ has a fixed point $y$ in $D$.

Theorem 3.2 In addition to the continuity of $\mathcal{F}: \mathbf{J} \times \mathbf{R} \times \mathbf{R} \rightarrow \mathbf{R}$, assume that there exists a positive constant $k$ such that for each $t \in \mathbf{J}$ and all $x, y, u, v \in \mathbf{R}$,

$|\mathcal{F}(t, x, y)-\mathcal{F}(t, u, v)| \leq k(|x-u|+|y-v|)$,

if

$\max \left\{2 \mathscr{G}^{*} k, \frac{2 k\left(\Gamma(\omega+1) \Gamma(3-\omega)+\Delta \xi(1-\zeta) \beta^{\omega}+\Delta(1-\zeta)+\Delta \zeta(1-\xi) \alpha^{\omega}\right)}{\Gamma(\omega+1) \Gamma(3-\omega)}\right\}<1$,

then the BVP (1) has a unique solution on $\mathbf{J}$.

Proof Let $y, \bar{y} \in \widetilde{C}(\mathbf{J}, \mathbf{R})$, then for each $t \in \mathbf{J}$, we have $\mid(N y(t))-(N \bar{y}(t) \mid$

$$
\begin{aligned}
\leq & \int_{0}^{1} \mid \mathscr{G}(t, \theta) \| \mathcal{F}\left(\theta, y(\theta),{ }^{c} \mathscr{D}^{\omega-1} y(\theta)\right) \\
& -\mathcal{F}\left(\theta, \bar{y}(\theta),{ }^{c} \mathscr{D}^{\omega-1} \bar{y}(\theta)\right) \mid \mathrm{d} \theta \\
\leq & \mathscr{G}^{*} k\left(\|y-\bar{y}\|_{\infty}+\left\|{ }^{c} \mathscr{D}^{\omega-1} y-{ }^{c} \mathscr{D}^{\omega-1} \bar{y}\right\|_{\infty}\right) \\
\leq & 2 \mathscr{G}^{*} k\|y-\bar{y}\|_{\widetilde{C}} .
\end{aligned}
$$

Thus

$\|N y-N \bar{y}\|_{\widetilde{C}} \leq 2 \mathscr{G}^{*}\|y-\widetilde{y}\|_{\widetilde{C}}$.

And thus (10) implies that $N$ is a contraction. Thanks to Banach contraction theorem, $N$ has a unique fixed point.

\section{Generalized Ulam-Hyers stability of the solutions of BVP (1)}

In this section, we prove necessary and sufficient conditions for the Ulam-Hyers (UHS) and generalized Ulam-Hyers

stability (GUHS) of the solutions to considered BVP (1) of nonlinear FODEs. To come across the required result, we give the following auxiliary results needed onward.

Definition 4.1 The solution $y \in \tilde{C}(\mathbf{J}, \mathbf{R})$ of the considered problem (1) is Ulam-Hyers stable(UHS) if we can find a real number $\hat{C}_{\mathcal{F}}>0$ with the property that for every $\varepsilon>0$ and for every solution $y \in \tilde{C}(\mathbf{J}, \mathbf{R})$ of the inequality

$\left.\left.\mid{ }^{c} \mathscr{D}^{\omega} y(t)\right)\right)-\mathcal{F}\left(t, y(t),{ }^{c} \mathscr{D}^{\omega-1} y(t)\right) \mid \leq \varepsilon, t \in \mathbf{J}$,

there exists unique solution $x \in \widetilde{C}(\mathbf{J}, \mathbf{R})$ of the proposed BVP (1) with a constant $\hat{C}_{\mathcal{F}}>0$ with

$\|x-y\|_{\widetilde{C}} \leq \hat{C}_{\mathcal{F}} \varepsilon$. 
Definition 4.2 The solution $y \in \widetilde{C}(\mathbf{J}, \mathbf{R})$ of the proposed BVP(1) is called to be generalized Ulam-Hyers stable (GUHS), if we can find

$\Theta_{\mathcal{F}}:(0, \infty) \rightarrow \mathbf{R}^{+}, \Theta_{\mathcal{F}}(0)=0$,

such that for each solution $y \in \widetilde{C}(\mathbf{J}, \mathbf{R})$ of the inequality (13), we can find a unique solution $x \in \widetilde{C}(\mathbf{J}, \mathbf{R})$ of the considered BVP (1) with

$\|x-y\|_{\widetilde{C}} \leq \hat{C}_{\mathcal{F}} \Theta_{\mathcal{F}}$.

Remark 4.3 A function $y \in \widetilde{C}(\mathbf{J}, \mathbf{R})$ is said to be the solution of inequality given in (13) if and only if, we can find a function $\hbar \in \widetilde{C}(\mathbf{J}, \mathbf{R})$ depends on $y$ only such that

(i) $|\hbar(t)| \leq \varepsilon$, for all $t \in \mathbf{J}$;

(ii) ${ }^{c} \mathscr{D}^{\omega} y(t)=\mathcal{F}\left(t, y(t),{ }^{c} \mathscr{D}^{\omega-1} y(t)\right)+\hbar(t)$, for all $t \in \mathbf{J}$.

Lemma 4.4 Under the assumption given as (9) and Remark 4.3, the solution $y \in \widetilde{C}(\mathbf{J}, \mathbf{R})$ of the BVP given by

$\left\{\begin{array}{l}{ }^{c} \mathscr{D}^{\omega} y(t)=\mathcal{F}\left(t, y(t),{ }^{c} \mathscr{D}^{\omega-1} y(t)\right)+\hbar(t), t \in \mathbf{J}=[0,1], \\ y(0)=\zeta y(\alpha), y(1)=\xi y(\beta),\end{array}\right.$

satisfies the relation given by

$\left|y(t)-\int_{0}^{1} \mathscr{G}(t, \theta) \mathcal{F}\left(\theta, y(\theta),{ }^{c} \mathscr{D}^{\omega-1} y(\theta)\right) \mathrm{d} \theta\right|$

$\leq \mathscr{G}^{*} \varepsilon$, for all $t \in \mathbf{J}$.

Proof Thanks to Lemma 2.5, we get the solution of BVP (14) as

$$
\begin{aligned}
y(t)= & \int_{0}^{1} \mathscr{G}(t, \theta) \mathcal{F}\left(\theta, y(\theta),{ }^{c} \mathscr{D}^{\omega-1} y(\theta)\right) \mathrm{d} \theta \\
& +\int_{0}^{1} \mathscr{G}(t, \theta) \hbar(\theta) \mathrm{d} \theta, t \in J,
\end{aligned}
$$

where $\mathscr{G}(t, \theta)$ is the same Green's function defined in Lemma 2.5. From (16), we may write as

$$
\begin{aligned}
\mid y(t) & -\int_{0}^{1} \mathscr{G}(t, \theta) \mathcal{F}\left(\theta, y(\theta),{ }^{c} \mathscr{D}^{\omega-1} y(\theta)\right) \mathrm{d} \theta \mid \\
& =\left|\int_{0}^{1} \mathscr{G}(t, \theta) \hbar(\theta) \mathrm{d} \theta\right| \\
& \leq \max _{t \in \mathbf{J}} \int_{0}^{1}|\mathscr{G}(t, \theta)||\hbar(\theta)| \mathrm{d} \theta \\
& \leq \mathscr{G}^{*} \varepsilon, \text { for all } t \in \mathbf{J} .
\end{aligned}
$$

Theorem 4.5 Under Assumption (9) and Lemma 4.4 together with the condition that $1 \neq 2 k\left(\mathscr{G}^{*}+\Lambda\right)$, the solutions of BVP (1) are Ulam-Hyers (UHS) and consequently generalized Ulam-Hyers stable(GUHS).

Proof Let $y \in \tilde{C}(\mathbf{J}, \mathbf{R})$ be any solution of BVP (1) and $x \in \tilde{C}(\mathbf{J}, \mathbf{R})$ be the unique solution of the considered problem (1), then consider

$$
\begin{aligned}
|y(t)-x(t)|= & \mid y(t)-\int_{0}^{1} \mathscr{G}(t, \theta) \mathcal{F}\left(\theta, y(\theta),{ }^{c} \mathscr{D}^{\omega-1} y(\theta)\right) \mathrm{d} \theta \\
& +\int_{0}^{1} \mathscr{G}(t, \theta) \mathcal{F}\left(\theta, y(\theta),{ }^{c} \mathscr{D}^{\omega-1} y(\theta)\right) \mathrm{d} \theta \\
& -\int_{0}^{1} \mathscr{G}(t, \theta) \mathcal{F}\left(\theta, x(\theta),{ }^{c} \mathscr{D}^{\omega-1} x(\theta)\right) \mathrm{d} \theta \mid \\
\leq & \left|y(t)-\int_{0}^{1} \mathscr{G}(t, \theta) \mathcal{F}\left(\theta, y(\theta),{ }^{c} \mathscr{D}^{\omega-1} y(\theta)\right) \mathrm{d} \theta\right| \\
& +\mid \int_{0}^{1} \mathscr{G}(t, \theta) \mathcal{F}\left(\theta, y(\theta),{ }^{c} \mathscr{D}^{\omega-1} y(\theta)\right) \mathrm{d} \theta \\
& -\int_{0}^{1} \mathscr{G}(t, \theta) \mathcal{F}\left(\theta, x(\theta),{ }^{c} \mathscr{D}^{\omega-1} x(\theta)\right) \mathrm{d} \theta \mid \\
\leq & \mathscr{G}^{*} \varepsilon+\int_{0}^{1}|\mathscr{G}(t, \theta)| k\left[|y-x|+\mid{ }^{c} \mathscr{D}^{\omega-1} y(\theta)\right. \\
& \left.-\mathscr{D}^{\omega-1} x(\theta) \mid\right] \mathrm{d} \theta,
\end{aligned}
$$

which on simplification like (11) yields that

$$
\begin{gathered}
\|y-x\|_{\infty} \leq \mathscr{G}^{*} \varepsilon+2 \mathscr{G}^{*} k\left[\|y-x\|_{\infty}\right. \\
\left.+\|\|^{c} \mathscr{D}^{\omega-1} y-{ }^{c} \mathscr{D}^{\omega-1} x \|_{\tilde{C}}\right] .
\end{gathered}
$$

Also we have

$$
\begin{aligned}
\left\|^{c} \mathscr{D}^{\omega-1} y-{ }^{c} \mathscr{D}^{\omega-1} x\right\|_{\infty} \leq 2 k \Lambda\|y-x\|_{\tilde{C}} & \\
\text { where, } \Lambda & =\frac{1}{\Gamma(\omega+1) \Gamma(3-\omega)}(\Gamma(\omega+1) \Gamma(3-\omega) \\
& \left.+\Delta \xi(1-\zeta) \beta^{\omega}+\Delta(1-\zeta)+\Delta \zeta(1-\xi) \alpha^{\omega}\right) .
\end{aligned}
$$

From Inequalities (17) and (18), we have

$\|y-x\|_{\widetilde{C}} \leq \mathscr{G}^{*} \varepsilon+2 k\left(\mathscr{G}^{*}+\Lambda\right)\|y-x\|_{\widetilde{C}}$.

which implies that

$\|y-x\|_{\widetilde{C}} \leq \frac{\mathscr{G}^{*} \varepsilon}{1-2 k\left(\mathscr{G}^{*}+\Lambda\right)}$. 
Hence, the solution of BVP (1) is Ulam-Hyers stable (UHS). Also if we let $\Theta(\varepsilon)=\varepsilon$ and $\tilde{C}_{\mathcal{F}}=\frac{\mathscr{G}^{*}}{1-2 k\left(\mathscr{G}^{*}+\Lambda\right)}$, then (19) can be written as

$\|y-x\|_{\widetilde{C}} \leq \widetilde{C}_{F} \Theta(\varepsilon)$.

It is clear that $\Theta(0)=0$. Hence, the solution of the proposed problem (1) is generalized Ulam-Hyers stable (GUHS).

Example 4.6 Consider the problem

$\left\{\begin{array}{l}{ }^{c} \mathscr{D}^{1.8} y(t)=\left(\frac{\cos t}{15 \mathrm{e}^{t}+2}\right)\left(\frac{1}{3+|y(t)|+\left|{ }^{c} \mathscr{D}^{0.8} y(t)\right|}\right), \quad t \in \mathbf{J}=[0,1], \\ y(0)=0.2 y(0.5), y(1)=0.075 y(0.75),\end{array}\right.$

where $\mathcal{F}(t, u, v)=\left(\frac{\cos t}{15 \mathrm{e}^{t}+2}\right)\left(\frac{1}{3+|u|+|v|}\right)$. Let $u, v, \bar{u}, \bar{v} \in \mathbf{R}$ and

$t \in \mathbf{J}$, then we have

$|\mathcal{F}(t, u, v)-\mathcal{F}(t, \bar{u}, \bar{v})| \leq \frac{1}{17}(|u-\bar{u}|+|v-\bar{v}|)$.

Hence, second condition of Theorem 3.2 holds with $k=\frac{1}{17}$. From (3), we have $\mathscr{G}^{*}<\frac{2.1079}{\Gamma(\omega+1)}$ for $\alpha=0.5, \beta=0.75, \zeta=0.2, \xi=0.075$ and $\Delta=1.18$. Now for $k=\frac{1}{17}$ and $\omega=1.8$, we have

\section{Conclusion}

In this paper we have considered a class of BVP of nonlinear FODEs. By using well-known Schauder's and Banach fixed point theorems, we have established some sufficient conditions for existence and uniqueness of solution to the considered problem. Since stability analysis is very important aspect of existence theory, we have also developed some results about Ulam-Hyers and generalized Ulam-Hyers type stability. For the demonstration of our analysis, we have given a suitable example. The established results generalize many results of the literature in two different ways. First of all we have taken four-point BVP of nonlinear FODEs instead of two-point BVP in our paper. Secondly, we have investigated two important forms of stability including Ulam-Hyers and generalized Ulam-Hyers type which have not been investigated for those BVP of FODEs in which nonlinear function depends on derivative term of dependent function (solution to be determined) to the best of our information. Further, our results generalize many results of the literature; we refer few of them as $[34,35]$.

Acknowledgements We are really thankful to the anonymous referee and editor for their valuable comments which improved this paper very well.

\section{Compliance with ethical standards}

Conflicts of interest It is declared that no competing interest exists regarding this manuscript.

$\frac{2 k}{\Gamma(\omega+1) \Gamma(3-\omega)}\left\{\Gamma(\omega+1) \Gamma(3-\omega)+\Delta \xi(1-\zeta) \beta^{\omega}+\Delta(1-\zeta)+\Delta \zeta(1-\xi) \alpha^{\omega}\right\}=0.2<1$

Thus

Authors contribution All authors have equal contribution to this manuscript.

$\max \left\{\frac{2}{17} \mathscr{G}^{*}, \frac{2\left(\Gamma(\omega+1) \Gamma(3-\omega)+\Delta \xi(1-\zeta) \beta^{\omega}+\Delta(1-\zeta)+\Delta \zeta(1-\xi) \alpha^{\omega}\right)}{17 \Gamma(\omega+1) \Gamma(3-\omega)}\right\}<1$,

By Theorem 3.2 it follows that the problem (20) has a unique solution on $\mathbf{J}$. Moreover $2 k\left(\mathscr{G}^{*}+\Lambda\right)=0.17145 \neq 1$. Hence, in view of Theorem 4.5 the solution of the BVP (20) is Ulam-Hyers stable and consequently generalized UlamHyers stable.
Open Access This article is distributed under the terms of the Creative Commons Attribution 4.0 International License (http://creativeco mmons.org/licenses/by/4.0/), which permits unrestricted use, distribution, and reproduction in any medium, provided you give appropriate credit to the original author(s) and the source, provide a link to the Creative Commons license, and indicate if changes were made. 


\section{References}

1. Podlubny, I.: Fractional Differential Equation. Academic Press, New York (1999)

2. Kilbas, A.A., Srivastava, H.M., Trujillo, J.J.: Theory and Applications of Fractional Differential Equations North-Holland Mathematics studies, vol. 204. Elsevier, Amsterdam (2006)

3. Benchohra, B., Graef, J.R., Hamani, S.: Existence results for boundary value problem with nonlinear fractinal differential equation. Appl. Anal. 87, 851-863 (2008)

4. Zhang, S.Q.: The existence of a positive solution for a nonlinear fractional differential equation. J. Math. Anal. Appl. 252, 804-812 (2000)

5. Belarbi, A., Benchohra, M., Ouahab, A.: Existence result for fractional differential equation of fractional order. Appl. Anal. 85, 1459-1470 (2006)

6. Bai, Z., Lu, H.: Positive solutions for boundary value problem of nonlinear fractional differential equation. J. Math. Anal. Appl. 311, 495-505 (2005)

7. Li, C.F., Luo, X.N., Zhou, Y.: Existence of positive solutions of the boundary value problem for nonlinear fractional differential equations. Comput. Math. Appl. 59, 1363-1375 (2010)

8. Babakhani, A., Gejji, V.D.: Existence of positive solutions of nonlinear fractional differential equations. J. Math. Anal. Appl. 278, 434-442 (2003)

9. Zhang, S.Q.: Existence of positive solution for some class of a nonlinear fractional differential equations. J. Math. Anal. Appl. 278, 136-148 (2003)

10. Bai, Z., Wang, Y., Ge, W.: Triple positive solutions for a class of two-point boundary-value problems. Electron. J. Differ. Equ. 6, $1-8$ (2004)

11. Ahmad, B., Nieto, J.J.: Existence of solutions for anti-periodic boundary value problems involving fractional differential equations via Leray-Schauder degree theory. Topol. Meth. Nonlinear Anal. 35, 295-304 (2010)

12. Shah, K., Khan, R.A.: Iterative scheme for a coupled system of fractional-order differential equations with three-point boundary conditions. Math. Methods Appl. Sci. 41(3), 1047-1053 (2018)

13. Makvand Chaharlang, M., Razani, A.: Existence of infinitely many solutions for a class of nonlocal problems with Dirichlet boundary condition. Commun. Korean Math. Soc. 34(1), 155-167 (2019)

14. Makvand Chaharlang, M., Razani, A.: Infinitely many solutions for a fourth order singular elliptic problem. Filomat 32(14), 50035010 (2018)

15. Mahdavi Khanghahi, R., Razani, A.: Solutions for a singular elliptic problem involving the $p(x)$-Laplacian. Filomat 32(14), 4841-4850 (2018)

16. Khalkhali, S.M., Razani, A.: Multiple solutions for a quasilinear (p, q)-elliptic system. Electron. J. Differ. Equ. 144, 1-14 (2013)

17. Khalkhali, S.M., Heidarkhani, S., Razani, A.: Infinitely many solutions for a fourth-order boundary-value problem. Electron. J. Differ. Equ. 164, 1-14 (2012)
18. Rehman, M., Khan, R.A.: Existence and uniqueness of solutions for multi-point boundary value problems for fractional differential equations. Appl. Math. Lett. 23, 1038-1044 (2010)

19. Khan, R.A., Rehman, M.: Existence of multiple positive solutions for a general system of fractional differential equations. Commun. Appl. Nonlinear Anal. 18, 25-35 (2011)

20. Khan, R.A., Rehman, M., Asif, N.: Three point boundary value problems for nonlinear fractional differential equations. Acta. Math. Sci. 31(B4), 1-10 (2011)

21. Rehman, M., Khan, R.A.: A note on boundary value problems for a coupled system of fractional differential equations. Comput. Math. Appl. 61, 2630-2637 (2011)

22. Benchohra, M., Naima Hamidi, J.Henderson: Fractional differential equations with anti-periodic boundary conditions. Numer. Funct. Anal. Optim. 34(4), 404-414 (2013)

23. Lijun, G., Wang, D., Wang, G.: Further results on exponential stability for impulsive switched nonlinear time-delay systems with delayed impulse effects. Appl. Math. Comput. 268, 186-200 (2015)

24. Stamova, I.: Mittag-Leffler stability of impulsive differential equations of fractional order. Q. Appl. Math. 73(3), 525-535 (2015)

25. Trigeassou, J.C., et al.: A Lyapunov approach to the stability of fractional differential equations. Signal Process. 91(3), 437-445 (2011)

26. Ulam, S.M.: Problems in Modern Mathematics. Wiley, New York (1940)

27. Ulam, S.M.: A Collection of Mathematical Problems. Interscience, New York (1960)

28. Obloza, M.: Hyers stability of the linear differential equation. Rocznik Nauk-Dydakt. Prace Mat. 13, 259-270 (1993)

29. Wang, J., Li, X.: Ulam Hyers stability of fractional Langevin equations. Appl. Math. Comput. 258, 72-83 (2015)

30. Kumama, P., Ali, A., Shah, K., Khan, R.A.: Existence results and Hyers-Ulam stability to a class of nonlinear arbitrary order differential equations. J. Nonlinear Sci. Appl. 10, 2986-2997 (2017)

31. Wang, J., Lv, L., Zhou, Y.: Ulam stability and data dependec for fractional differential equations with Caputo derivative. Electron. J. Qual. Theory Differ. Equ. 63, 1-10 (2011)

32. Haq, F., Shah, K., Rahman, G., Shahzad, M.: Hyers-Ulam stability to a class of fractional differential equations with boundary conditions. Int. J. Appl. Comput. Math. 2017, 1-13 (2017)

33. Mokhtarzadeh, M.R., Pournaki, M.R., Razani, A.: Note on periodic solutions of Riccati equations. Nonlinear Dyn. 62, 119-125 (2010)

34. Khan, R.A.: Three-point boundary value problems for higher order nonlinear fractional differential equations. J. Appl. Math. Inform. 31(12), 221-228 (2013)

35. Bai, Z.: On positive solutions of a nonlocal fractional boundary value problem. Nonlinear Anal. 72, 916-924 (2010)

Publisher's Note Springer Nature remains neutral with regard to jurisdictional claims in published maps and institutional affiliations. 